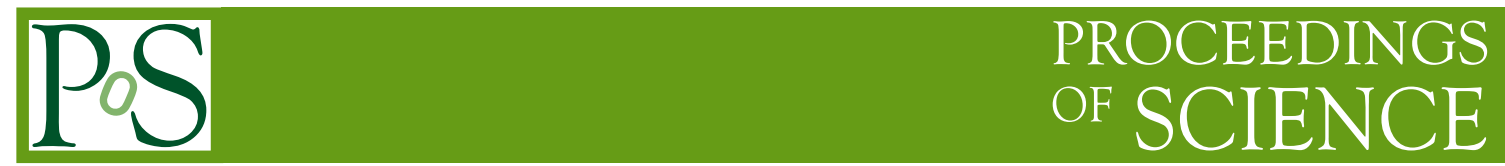

\title{
Atlas Silicon Strips Operations and Performance
}

\section{Urban Bitenc*}

Physikalisches Institut-Abert-Ludwigs-Universitaet

E-mail: urban.bitencephysik.uni-freiburg.de

\begin{abstract}
The current status of the ATLAS SemiConductor Tracker is presented. We report on the operation of the detector and include results from the latest data-taking periods in autumn 2009 and spring 2010. The collected data is compared to the design requirements and to predictions of Monte Carlo simulation. This shows a very good understanding and a very good performance of the detector.
\end{abstract}

19th International Workshop on Vertex Detectors - VERTEX 2010

June 06 - 11, 2010

Loch Lomond, Scotland, UK

${ }^{*}$ Speaker. 


\section{Introduction}

ATLAS [1] is a general purpose detector at the Large Hadron Collider (LHC) at CERN, Geneva. The LHC is designed to collide protons at a centre of mass energy of $\sqrt{s}=14 \mathrm{TeV}$ and a luminosity of $10^{34} \mathrm{~cm}^{-2} \mathrm{~s}^{-1}$. The total cross section at the LHC is expected to be roughly $100 \mathrm{mb}$ [2]. The unprecedented energy and luminosity of the LHC imposes tight constraints and requirements on the layout of the detectors: high granularity, fast readout and a high radiation hardness are needed. The Inner Detector (ID), the innermost tracking device of ATLAS, is located in a $2 \mathrm{~T}$ solenoidal magnetic field. The ID consists of a silicon pixel detector, a silicon strip detector and a straw tube transition radiation tracker (TRT). Its main design goals are the primary and secondary vertex identification as well as a precise momentum measurement of charged particles in the region $|\eta|<2.5$.

\section{The SemiConductor Tracker}

The silicon strip detector is called SemiConductor Tracker (SCT) and is located between the Pixel and TRT detectors. As can be seen in Figure 1, it consists of a barrel and two endcap detectors. The barrel is made of four cylindrical layers containing 2112 modules. Each of the endcaps is made of nine radial disks containing in total 988 modules. Depending on the particular layer, the barrel covers $|\eta|<1.1$ to 1.4 , and the endcaps cover the region up to $|\eta|<2.5$.

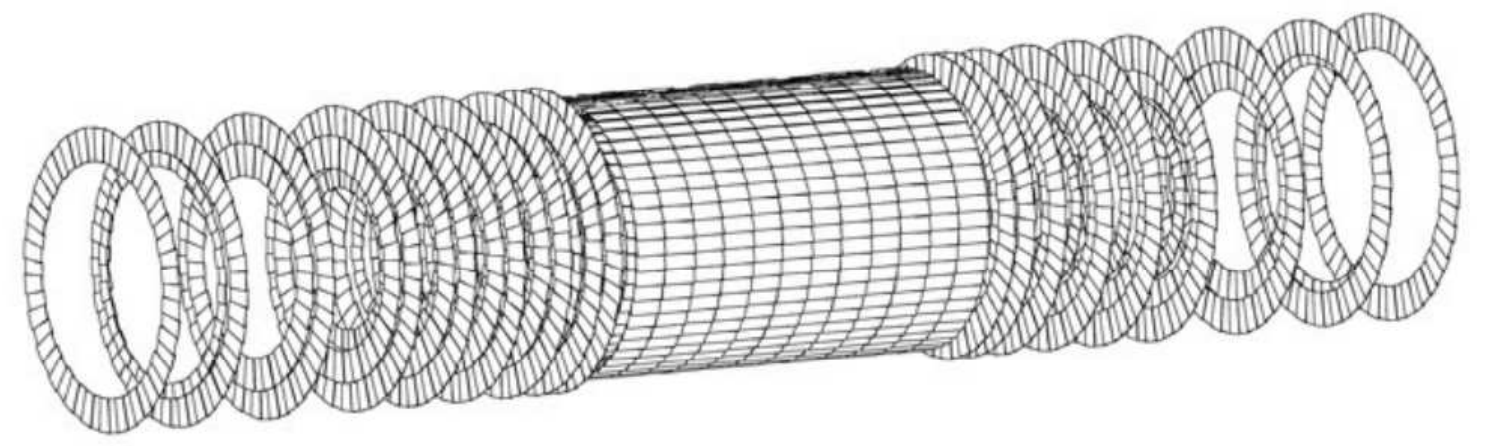

Figure 1: The ATLAS SCT detector: 9 discs in each of the endcaps and 4 layers in the barrel.

The SCT modules [3, 4] consist of two sensor pairs, glued back-to-back and rotated by a stereo angle of $40 \mathrm{mrad}$ with respect to each other. The sensors are constructed using p-type readout strips on n-type bulk silicon. The strip pitch is $80 \mu \mathrm{m}$ in the barrel and varies in the endcaps between $57 \mu \mathrm{m}$ and $94 \mu \mathrm{m}$ due to a fan geometry. The strip length is $12.6 \mathrm{~cm}$ in the barrel and $12.1 \mathrm{~cm}$, $11.7 \mathrm{~cm}$ and $7.2 \mathrm{~cm}$ in the end-caps (for the three different layouts). There are 768 active readout channels per module side. A binary readout with a default threshold of $1 \mathrm{fC}$ is provided by six radiation hard $\mathrm{ABCD}$ chips [5] for one module side. For the module communication with the offdetector electronics optical links [6] are used. The conversion of the electronic to optical signal is done via laser diodes (VCSELs) [6]. The modules are operated at reverse bias with an initial voltage of $150 \mathrm{~V}$. For cooling an evaporative cooling system [7] is used with $\mathrm{C}_{3} \mathrm{~F}_{8}$ operating at the nominal temperature of $-25^{\circ} \mathrm{C}$. 
Tight requirements are imposed on the performance of the modules. The single hit resolution is required to be $17 \mu \mathrm{m}$ in the $\mathrm{R}-\phi$ and $580 \mu \mathrm{m}$ in the $\mathrm{z}$ direction, therefore imposing maximum module-to-module alignment tolerances of $12 \mu \mathrm{m}$ and $50 \mu \mathrm{m}$, respectively. Considering a 10 year operation period the detector is required to withstand a particle fluence of $2 \times 10^{14} \mathrm{Neq} \mathrm{cm}^{-2}$. Throughout this period the noise occupancy is required to be smaller than $5 \times 10^{-4}$ for a hit efficiency of $99 \%$, and at least $99 \%$ of the detector should be in operation.

The SCT detector is equipped with an optical alignment system, based on a Frequency Scanning Interferometry method [9], which is able to monitor movements of the SCT modules in real time up to a precision of a few $\mu \mathrm{m}$. More details about the alignment of the SCT are given in [10].

\section{Operation experience}

During 2009 and 2010, the SCT was very successfully used to collect data from proton-proton collisions. On November $23^{\text {rd }} 2009$ the first $900 \mathrm{GeV}$ collision data were collected with no magnetic field. The reverse bias voltage was set to $50 \mathrm{~V}$. On December $6^{\text {th }}$ first $900 \mathrm{GeV}$ collisions ware collected with stable beams and with the solenoidal field; the reverse bias voltage was set to the default value of $150 \mathrm{~V}$. Since March $30^{\text {th }} 2010$ large amounts of $7 \mathrm{TeV}$ collision data were collected with the SCT. In the absence of the stable beam flag the reverse bias voltage is kept at 20 V. Tables 1 and 2 summarise the status of excluded modules, chips and strips in the first half of 2010. More than $99 \%$ of the SCT is fully functional.

\begin{tabular}{lccccc}
\hline \hline Disabled Readout Components & Endcap A & Barrel & Endcap C & SCT & Fraction (\%) \\
\hline Disabled Modules & 5 & 10 & 15 & 30 & 0.73 \\
Disabled Chips & 5 & 24 & 4 & 33 & 0.07 \\
Masked Strips & 3,364 & 3,681 & 3,628 & 10,673 & 0.17 \\
\hline Total Disabled Detector Region & & & & & 0.97 \\
\hline \hline
\end{tabular}

Table 1: The table shows the summary of excluded modules, chips and masked strips for the ATLAS SCT in 2010. There is no overlap between modules and chips. The 33 excluded chips comprise 31 chips which are bypassed and 2 chips for which all strips are masked. The number of masked strips does not include strips from the two chips which are fully masked, but does include a small number of masked strips from other disabled chips. In total $0.97 \%$ of the SCT detector is disabled.

The SCT endcaps are operated at the silicon surface temperature of $-7^{\circ} \mathrm{C}$. The inner three barrel layers are operated at $-2^{\circ} \mathrm{C}$ to $-1.5^{\circ} \mathrm{C}$ and the outer one at $+4.5^{\circ} \mathrm{C}$. In 2009 a reassessment of cooling requirements and effects of radiation damage was made. The radiation damage as a function of the depletion voltage and leakage current is now expected to be smaller than predicted at the time of TDR [8].

The SCT is currently running in a so-called any-hit mode in which a strip is recorded as a hit if its signal is above the threshold in the triggered bunch crossing (BC) or in the $\mathrm{BC}$ before or after that of the trigger. An alternative readout mode for a higher luminosity is called level-sensing mode with a "x1x" pattern, meaning that a signal over threshold is required in the triggered BC. At a very high luminosity the SCT will run in the edge mode with a " $01 \mathrm{x}$ " pattern: a hit required in 


\begin{tabular}{lccccc}
\hline \hline & Endcap A & Barrel & Endcap C & SCT & Fraction (\%) \\
\hline Total & 5 & 10 & 15 & 30 & 0.73 \\
Fraction (\%) & 0.5 & 0.2 & 1.5 & 0.7 & \\
\hline Cooling & 0 & 0 & 13 & 13 & 0.32 \\
LV & 0 & 6 & 1 & 7 & 0.17 \\
HV & 4 & 1 & 1 & 6 & 0.15 \\
Readout & 1 & 3 & 0 & 4 & 0.10 \\
\hline \hline
\end{tabular}

Table 2: The table gives more details on the location and reasons for excluded modules. The 13 modules in Endcap $\mathrm{C}$ that are excluded due to cooling problems, all lie on one leaking cooling loop. LV and HV refers to the fact that modules need to be disabled due to a general problem related to either Low Voltage or High Voltage; the failures are not caused by a defective LV/HV card. "Readout" refers to a general problem with the optical communication.

the triggered $\mathrm{BC}$ and vetoed in the $\mathrm{BC}$ before the triggered one. A quantity that gives a measure on how well the SCT is timed-in is the average time bin of the hits. For each hit it is checked in which of the three time bins (before the triggered BC, in the triggered BC or after it) the signal was above the threshold. The time bins are assigned values of 0.0, 1.0 and 2.0 and the average value over a large number of hits is calculated. This quantity is shown in Figure 2. One can see, that the average time bin in the current any-hit mode is close to 1.0. This means that the SCT is well timed-in with the ATLAS detector, since the signal mostly arrives in the middle time bin. This also means that the SCT is ready to move to the level-sensing mode and edge mode when necessary.

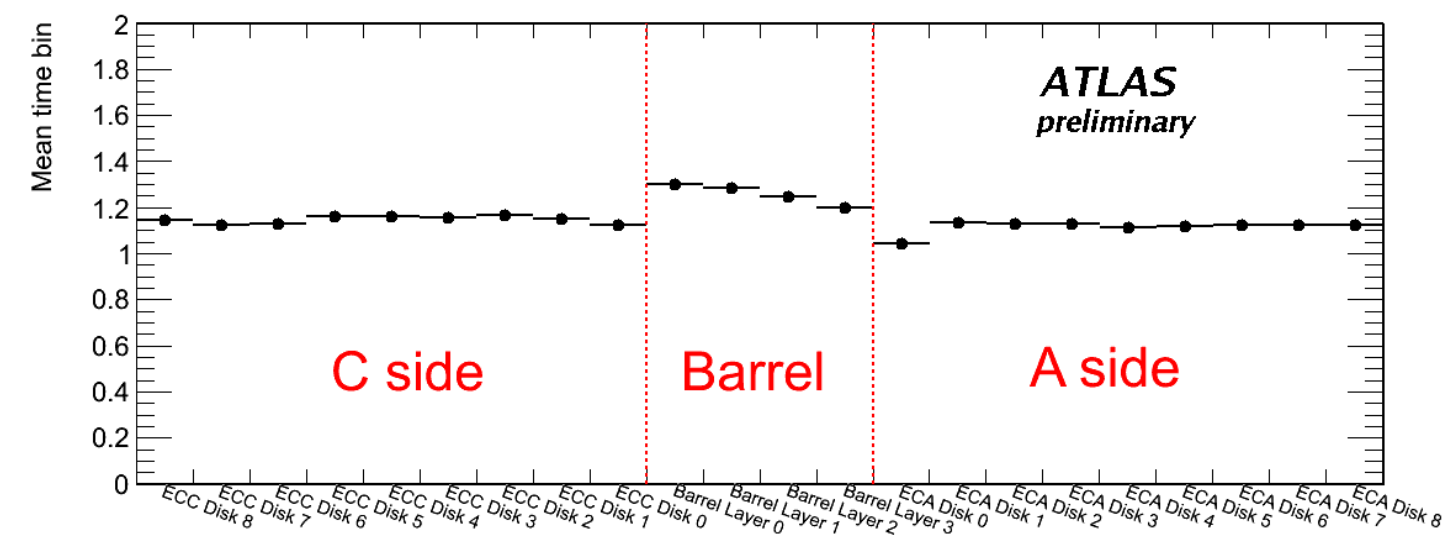

Figure 2: The average time bin for each of the barrel layers and endcap discs. The average is close to 1.0 which shows that the ATLAS SCT is well timed-in.

For the operation of the SCT detector there is one shifter in the ATLAS Control Room. For the data quality monitoring there is a remote shifter, checking efficiencies, noise, hit maps, timing, residuals, pulls and other characteristic distributions. Apart from that there are several shifters on-call.

Two biggest concerns with the operation of SCT are the evaporative cooling and the optical 
transmitters of clock and commands to the modules. In ATLAS the Pixel and SCT detectors share the cooling system. The system had many problems in years 2005-2008. In 2009 and 2010 only low level problems persist especially with oil-free, 2-stage, leak-less compressors. Four out of seven compressors are used for the low radiation damage period. For the future two scenarios are being studied. The first one would be to replace the compressors. The second one would be to reduce the compression ratio using gravity: take the vapor to the surface (about $85 \mathrm{~m}$ above the ATLAS detector), condense it and make use of the hydrostatic pressure of liquid. The second problem concerns the optical transmitters of clock and command signals. During the SCT commissioning, many of these laser diode transmitters failed. As a result, all were replaced prior to the first protonproton collisions. However, after a pause of six months the failures have continued, and detailed investigations are ongoing.

\section{Performance with first data}

In the presence of a magnetic field the drift path of the charge carriers inside a silicon detector is deflected due to the Lorentz force. The resulting Lorentz angle is an important quantity that the track finding algorithms have to take into account. The left plot of Figure 3 shows the average cluster size as a function of incidence angle for the four barrel layers in the $900 \mathrm{GeV}$ collision data. The minima of the distributions are as expected displaced from zero. The Lorentz angle is roughly determined as the incidence angle at the minimal average cluster size. The right plot of Figure 3 shows the fitted value of the Lorentz angle for the cosmic data runs and for the first two $900 \mathrm{GeV}$ runs with the SCT at the nominal settings, compared to the model prediction. They are in a good agreement.
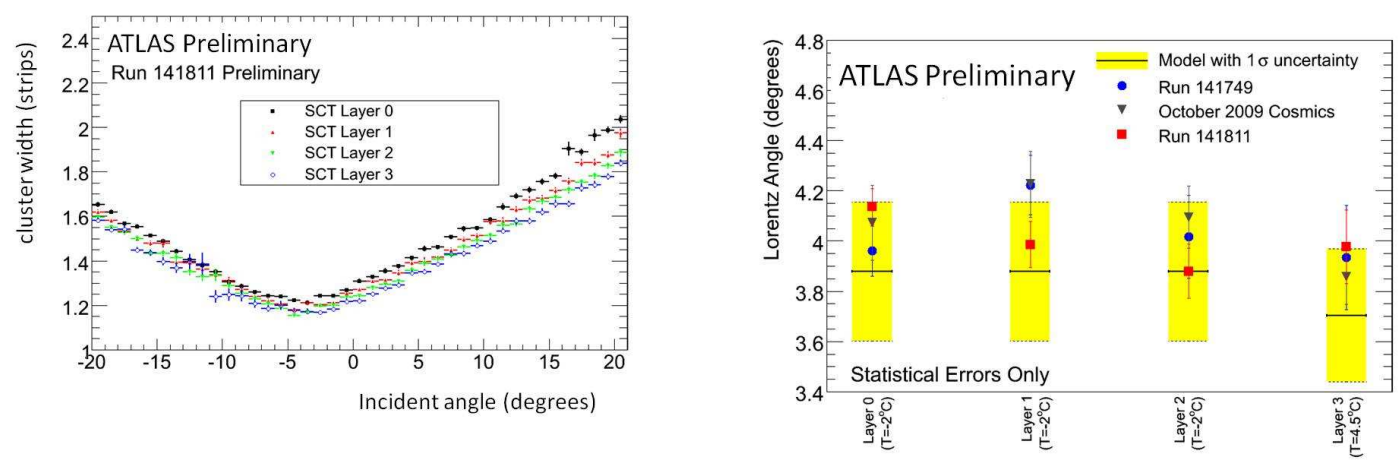

Figure 3: The left plot shows the average cluster size (number of strips hit) as a function of the incident track angle. The minimum of the distribution is at the Lorentz angle. The right plot shows the fitted value of the Lorentz angle for the cosmic data runs (black triangles) and for the first two $900 \mathrm{GeV}$ runs with the SCT at the nominal settings (red squares and blue circles), compared to the model prediction (yellow band).

Figure 4 shows the number of hit strips per module side in proton-proton collisions with $\sqrt{s}=$ $900 \mathrm{GeV}$. One can see that the average occupancy is low: typically only a couple of strips per 
module side are hit. Data is compared to Monte Carlo events and a good agreement is found for a wide range of number of hit strips.

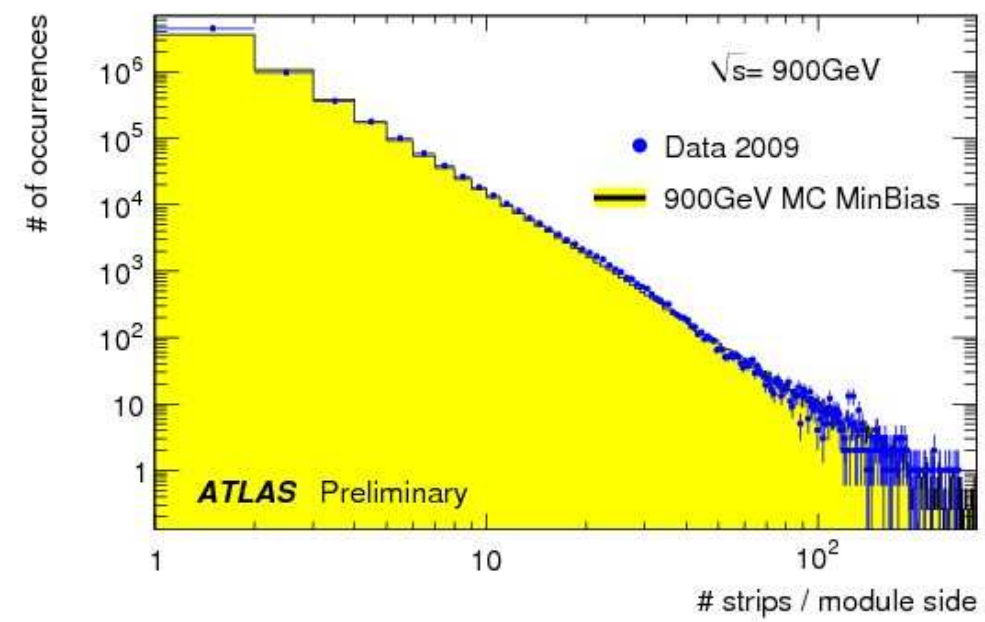

Figure 4: This plot shows the number of strips hit per module side in the $900 \mathrm{GeV}$ data and in the $900 \mathrm{GeV}$ MC events. The data and the simulation are normalised to the same number of events. A good agreement is obtained for a wide range of number of strips. The discrepancy in the first bin arises (at least in part) from the fact that the data were collected in any-hit mode, whereas for the Monte Carlo simulation the level-sensing mode was used and the noise is accordingly about three times lower. The difference between the two modes is explained in the text.

The intrinsic module efficiency was extracted using reconstructed tracks. A hit is expected on a module if a track traverses a module sensor ( $3 \sigma$ away from the border) and the module and chip are not excluded from the configuration (the excluded strips were not taken into account). The number of found hits divided by the number of expected hits gives the intrinsic module efficiency which is on average about $99.7 \%-99.8 \%$. Since the excluded individual strips are not taken into account and therefore contribute to inefficiency, the actual intrinsic module efficiency is even slightly higher. Two kinds of tracks were used: tracks reconstructed from the SCT hits only and tracks reconstructed using the whole Inner Detector information (Pixel hits, SCT hits and TRT hits). The results obtained using both kinds of tracks agree reasonably well.

The left plot of Figure 5 shows the distribution of input noise values, obtained in a so-called response curve test. For a given injected charge, the occupancy versus threshold is measured and parametrised by a fit using a complementary error function (the so-called S-curve). The width of the distribution is a measure of the noise. The typical value of 1500 electrons is well below the threshold of $1 \mathrm{fC}$ (6240 electrons). The right plot of Figure 5 shows the noise occupancy obtained using a random trigger. The mean of the distribution is well below the specification value of $5 \times 10^{-4}$.

\section{Conclusions}

During 2009 and 2010, proton-proton collision have been collected using the ATLAS SCT 

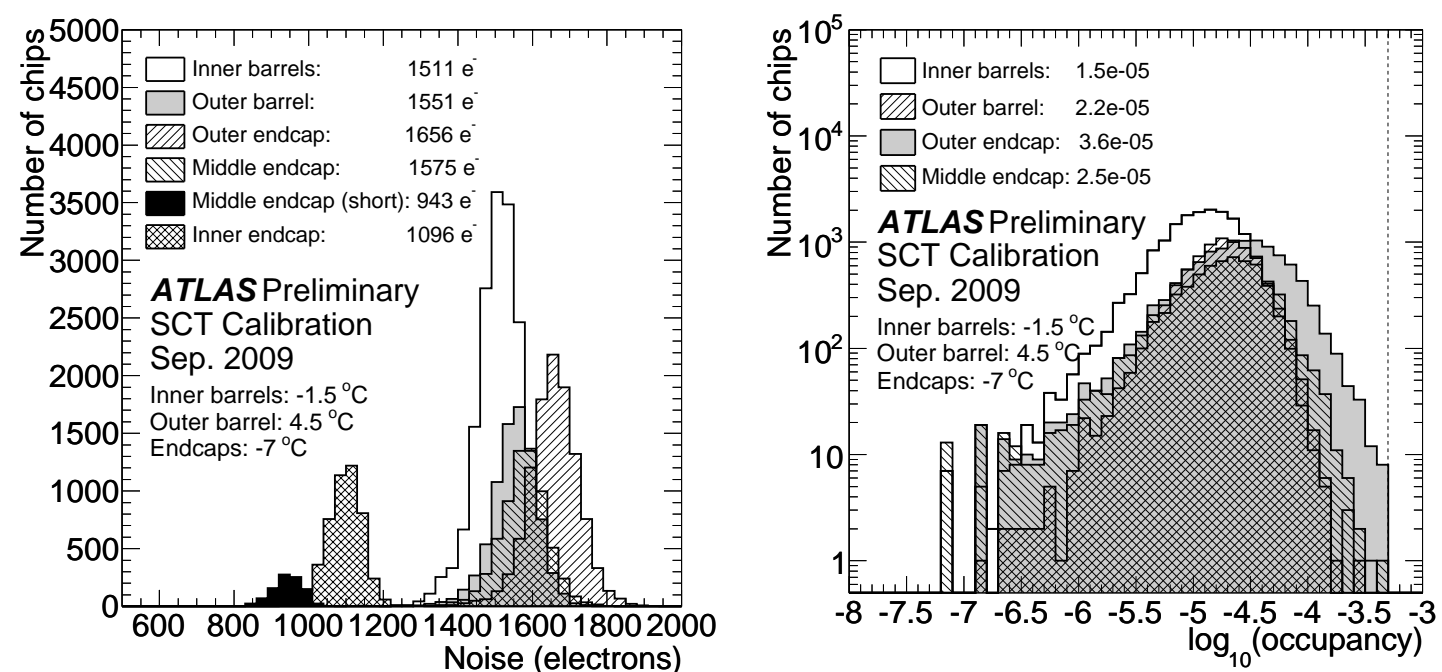

Figure 5: The left plot shows the distribution of input noise values for each chip in the different parts of the SCT. The results are extracted from the response curve tests. The right plot shows the noise occupancy obtained using a random trigger, averaged over chips in barrel and endcap detectors. The dashed line indicates the specification limit of $5 \times 10^{-4}$. The values for chips on the inner and middle short endcap modules are not shown since in the corresponding channels the average noise occupancy lies below the sensitivity of the performed tests. The average values for each detector region are given together with the estimated silicon temperature for the operation condition.

detector. Its operation and performance have been very successful. More than $99 \%$ of the SCT is fully operational. The measured intrinsic efficiency is above $99.7 \%$ and the average noise occupancy is well below $5 \times 10^{-4}$. All these values are within the specification requirements (which are set for an irradiated detector). Comparisons with simulation show a very good understanding of the detector.

\section{References}

[1] The ATLAS Collaboration, The ATLAS experiment at the CERN Large Hadron Collider, JINST 3 (2008) S08003

[2] A. Moraes, C. Buttar and I. Dawson, Prediction for minimum bias and underlying event at LHC energies, Eur. Phys. J., C 50 (2007) 435-466,

[3] A. Abdesselam et al., The barrel modules of the ATLAS semiconductor tracker, Nucl. Instrum. Meth. A 568 (2006) 642

[4] A. Abdesselam et al., The ATLAS semiconductor tracker end-cap module, Nucl. Instrum. Meth. A 575 (2007) 352

[5] F. Campabadal et al., Design and performance of the ABCD3TA ASIC for readout silicon strip in the ATLAS semiconductor tracker, Nucl. Instrum. Meth. A 552 (2005) 561

[6] A. Abdesselam et al., The optical links of the ATLAS Semi Conductor Tracker, JINST 2 (2007) P09003 
[7] D. Attree, B. Anderson, E. C. Anderssen, et al., The evaporative cooling system for the ATLAS inner detector, JINST 3 (2008) P07003

[8] Inner Detector Techical Design Report, ATLAS TDR 4, CERN/LHCC/97-16, ISBN 92-9083-102-2

[9] S. Gibson et al., Coordinate measurement in 2-D and 3-D geometries using frequency scanning interferometry, Opt. Laser Eng. 44 (2005) 79-95.

[10] G. Piacquadio, ATLAS Alignment, Tracking and Physics Performance Results, Proceedings at the Vertex 2010 Workshop (2010). 